\title{
Development of EOS data for granular material like sand by using micromodels
}

\author{
M. Larcher ${ }^{\mathrm{a}}$ and N. Gebbeken \\ Universität der Bundeswehr München, 85577 Neubiberg, Germany
}

\begin{abstract}
Detonations in soil can occur due to several reasons: e.g. land mines or bombs from the Second World War. Soil is also often used as a protective barrier. In all cases the behaviour of soil loaded by shock waves is important. The simulation of shock wave loaded soil using hydro-codes like AUTODYN needs a failure model as well as an equation of state (EOS). The parameters for these models are often not known. The popular material law for sand from Laine and Sandvik [1], e.g., is a first approximation, but it can only be used for dry sand with a certain grain grading. The parameters porosity, grain grading, and humidity have a big influence on the material behaviour of cohesive soils. Micro-mechanic models can be used to develop the material behaviour of granular materials. EOS data can be obtained by numerically loading micro-mechanically modelled grains and measuring the density under a certain pressure in the finite element model. The influence of porosity, grain grading, and humidity can be easily investigated. EOS data are determined in this work for cohesive soils depending on these parameters.
\end{abstract}

\section{Introduction}

The simulation of shock wave loaded soil is complex. Soil is often not a homogeneous material. Cohesive and noncohesive soils are often mixed; the diameters of the particles are varying and also other parameters like humidity have a significant influence on the behaviour of the soil. The numerical simulation of statically loaded noncohesive, granular soils is done using a shear strength as failure limit (e.g. Mohr-Coulomb).

The description of high strain rate loaded materials is done by splitting the stresses in a hydrostatic and a deviatoric part. The deviatoric part describes the failure of the material under shear stresses. Several failure surfaces can be adapted. The strain rate effect should be considered for the deviatoric part (e.g. for concrete Gebbeken [2]).

The hydrostatic part describes here the behaviour under high compressive strains. The influence of micro pores is very important. The behaviour can be described using so called equations of states (EOS) where pressuredensity functions are implemented. Another possibility is a so called Y-function (Larcher [3]). For some materials the pressure-density functions are known. The pressuredensity curve for steel is nearly linear since the porosity of steel is very small. The pressure-density curve for concrete is, however, more complex and is similar to the one of granular materials (Figure 1). The behaviour of concrete under hydrostatic load is affected by the behaviour of the micro-pores. Following a linear elastic hydrostatic loading the micro-pores fail (plastic loading). The compression stiffness decreases. Further compaction of the micro pores results in increased stiffness.

For granular materials (Figure 1) the EOS starts at the unloaded density $\rho_{0}$. This density corresponds to the density under low pressure compaction (e.g. gravity). The elastic part of the EOS is often very short. In the following part the pressure-density curve increases exponentially.

a e-mail: martin.larcher@unibw.de
For higher densities the curve converges to the EOS of the particle material.

The pressure-density curve of granular material like sand or gravel is often not known in detail. In the hydrocode AUTODYN mainly the 'sand' model from Laine and Sandvik [1] is used for all kind of granular soils. This model is based on some experiments of sand from Sjöbo, Norway and represents a quite coarse gravel when humidity most probably has no influence. The experiments are done using tri-axial cell devices. Some data for a fine cohesive soil (prairie soil) depending on the humidity is given by Fiserova [4]. The influence of the humidity of this soil is large. Investigations for STANAG 4569 sandy gravel are done by Grujicic [5], [6]. Partial formulas are given.

Experimental investigations in the field of shock wave loaded gravel materials are complex. Therefore, numerical models could be used here instead. Mesomechanical models are used successfully for concrete (Hartmann [7], Riedel [8]) by modelling separately the aggregates, the cement and the contact zone between these two. A micromechanical model of sand is built here using spheres of different diameters that are randomly arranged using a preprocessor. The spheres are represented by several finite elements. An adapted contact formulation is essential. The model is loaded hydrostatically. The displacements are used to obtain the density; the lateral forces are used to obtain the pressure. The combination results in the EOS.

\section{Properties of sand and gravel}

Sand and gravel are three-phase media containing solid particles, water and air. The moisture content can be expressed by the ratio of the amount of water and the mass of dry particles.

The solid part is described by the particle size distribution or grading. Several standards are available to classify the soil (DIN 18196, EN 12620). The particle size 


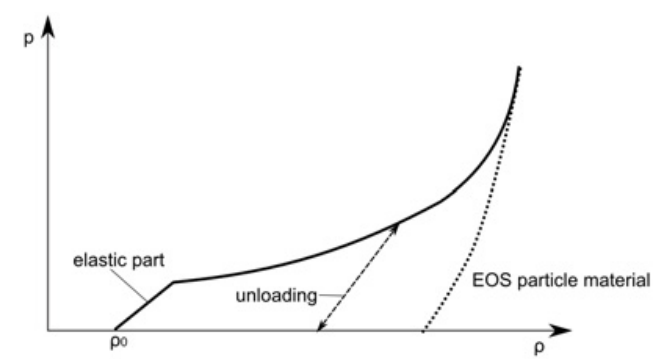

Fig. 1. Equation of state for granular materials.

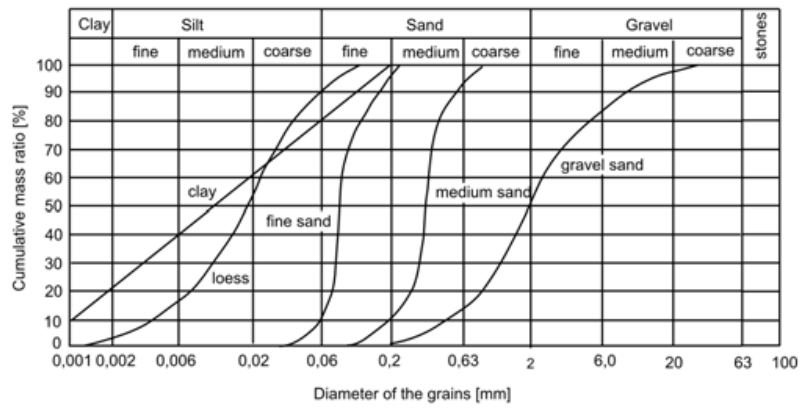

Fig. 2. Grain grading.

distribution is often illustrated using diagrams shown in Figure 2.

The initial density of the sand or gravel depends on the compaction of the material. The humidity of the sand has also an influence on the density but also on the compaction ability.

\section{Simulation model}

The micro-mechanical simulation model is performed in several steps. First, the development of the FE model using a preprocessor. Next, the simulation with the explicit finite element code LS-DYNA and last the determination of the pressure-density curve.

\subsection{Preprocessor}

The objective of the preprocessor is to create a finite element mesh containing several spheres randomly distributed. The preprocessor is written in FORTRAN since the development of the model is time consuming. Several input parameters have to be given: e.g. the size of the virtual box where the grains should be stored, the grain grading, the maximum number of grains.

The preprocessor is started using a drop-down procedure as shown in Figure 3. According to this grain grading the diameter of the next grain is determined in the way that it fulfils best the grain grading. This grain is let fall from the highest point at the randomly chosen position of the virtual box. The contact is controlled to other grains and to the sides of the box. If the grain stops at the ground of the box the next grain is chosen. If the grain falls onto another grain, the grain rolls off to the side of the grain and the drop-down process goes on from that grain position.

After that procedure the grains do not have the smallest possible package. Therefore, after passing a certain number of grains the virtual box is virtually turned to one of

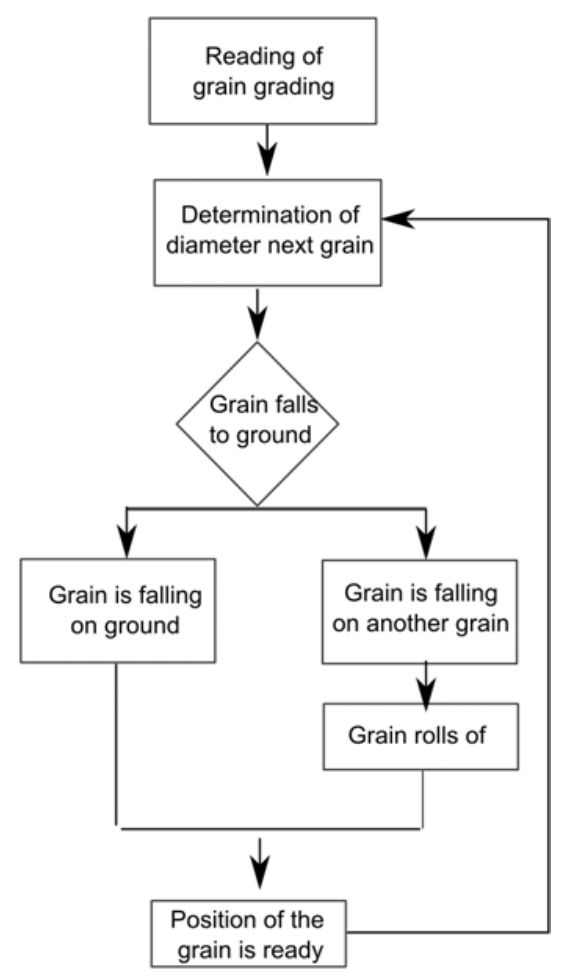

Fig. 3. Drop-down procedure of the preprocessor.

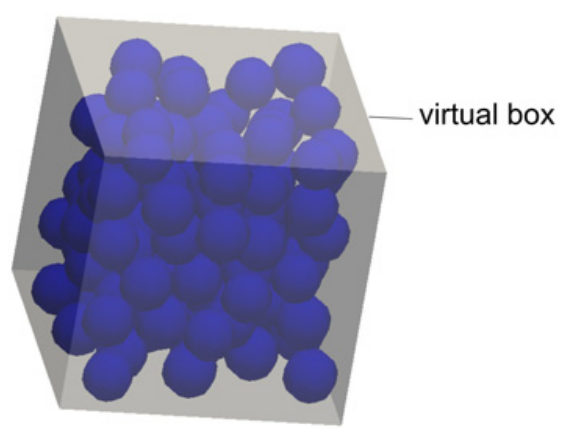

Fig. 4. Drop-down procedure: result.

the sides. The drop-down procedure is started again for all grains. This is done for all sides of the virtual box until no grain is moving any more. The drop-down procedure is repeated until the maximum of grains is reached. At the end, the diameter and the location of all spheres are known. A result of a preprocessor calculation until this step is shown in Figure 4.

During the last step the spheres are discretised by finite elements. For the calculations shown here, a sphere is split into 189 or 2000 finite elements respectively depending on the size of the sphere.

\subsection{Material parameters, simulation parameters}

The material parameters for the spheres are chosen using an elastic material with the density of $2730 \mathrm{~kg} / \mathrm{m}^{3}$ and a Young's modulus of $70 \mathrm{GPa}$. The Poisson's ratio is chosen to 0.3 . This material corresponds to the behaviour of granite. The failure of the material is not considered here since erosion would eliminate mass from the model. 


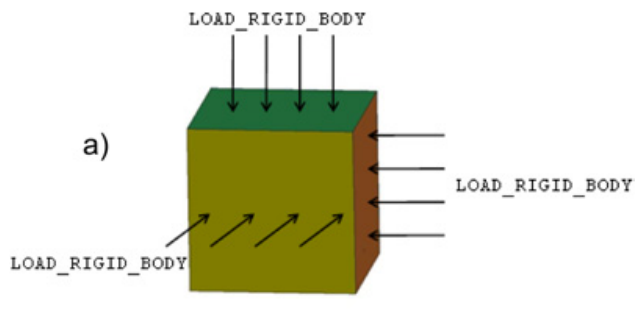

b)

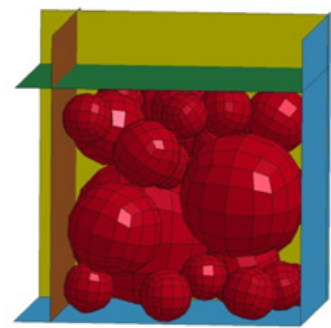

Fig. 5. Loading of the model, displaced configuration.

This would lead to unrealistic pressure-density curves. The failure of the material could be implemented by using non mass erosion failure procedures (e.g. node splitting).

The contact between the grains is very important. Here, the contact algorithm CONTACT _ AUTOMATIC_ SURFACE_ TO_ SURFACE shows a good convergence. The influence of the gravity is neglected.

The virtual box is modelled using rigid bodies. Three sides are set fixed, the other three sides are loaded uniformly in the normal direction by a constant speed (Figure 5(a)). Due to the loading, the grains are rearranged under the given loading so that the configuration of Figure 5(b) is reached at a certain point.

\subsection{Determination of the pressure-density curve}

The pressure-density curve is obtained using the following procedure: The rigid body displacement results in a changed volume. Since the mass of the model is not changed the density can be calculated by the ratio between mass and remaining volume for a given time step. The forces at the rigid bodies of the virtual box sides can be used to obtain the pressure at a given time step.

\section{Results}

\subsection{Model with 110 particles}

The first calculation is performed using 110 particles represented by 47494 finite elements. The elements are reduced constant stress solid elements. The target grading of the particles is given in Table 1. The particles are located in a cubical virtual box of $1 \mathrm{~cm}^{3}$. The initial density was $874.7 \mathrm{~kg} / \mathrm{m}^{3}$. Figure 6 shows the model at three loading steps $\left(t_{1}=0 \mathrm{~s}, t_{2}=0.001 \mathrm{~s}, t_{3}=\right.$ $0.002 \mathrm{~s}$ corresponding to a density of $\rho_{1}=874.7 \mathrm{~kg} / \mathrm{m}^{3}$, $\left.\rho_{2}=1504.0 \mathrm{~kg} / \mathrm{m}^{3}, \rho_{3}=1526.1 \mathrm{~kg} / \mathrm{m}^{3}\right)$. The compaction until a density of about $1460 \mathrm{~kg} / \mathrm{m}^{3}$ occurs fast. The pressure that is necessary to reach this density is small (Figure 7). This density corresponds with the unloaded density of sand. Beginning from that point, the pressure
Table 1. Target grading for models with 110,200 and 25 particles.

\begin{tabular}{ll}
\hline Size $[\mathrm{mm}]$ & Part [\%] \\
\hline $1-1.5$ & 7.4 \\
$1.5-2$ & 7.4 \\
$2-3$ & 14.9 \\
$3-5$ & 22.3 \\
$5-7$ & 22.3 \\
$7-12$ & 25.5 \\
\hline
\end{tabular}
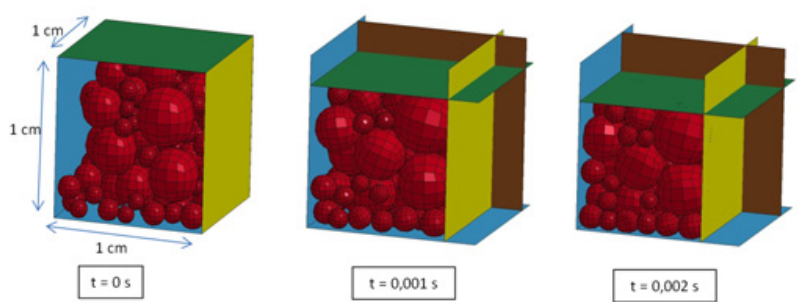

Fig. 6. Displacements of the spheres under hydrostatical loading.

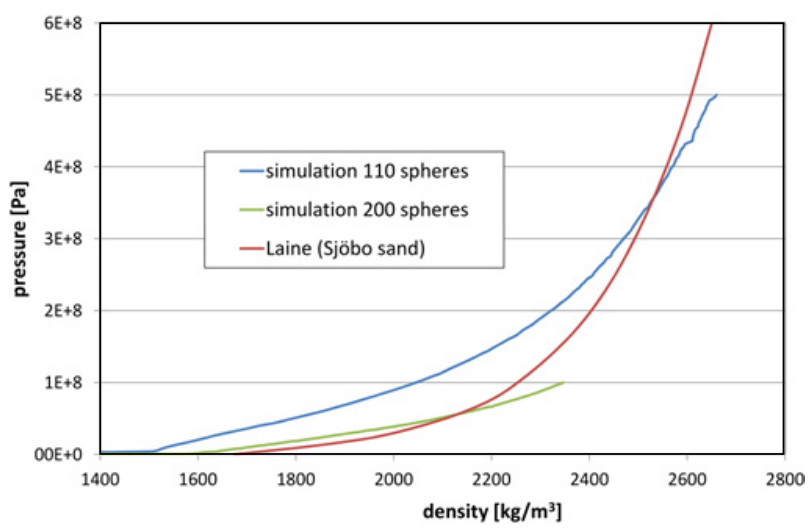

Fig. 7. Pressure-density curve of the numerical experiment (110 and 200 spheres). Comparison with the data from Laine [1].

increases significantly. The pressure-density curve shows the characteristic exponential increase of the pressure as expected for granular materials. The comparison with the pressure-density curve from Laine [1] shows a good accordance taking into account that the grain grading is not given in detail.

It is important to note that the curve from Laine is experimentally validated until a pressure of about $60 \mathrm{MPa}$. All values above are extrapolated. Therefore, this data should not be used for validation purposes.

\subsection{Model with 200 particles}

The model with 200 particles (90048 elements) shows a similar behaviour. The size of the virtual box was chosen to be $8 \mathrm{~cm}^{3}$. The initial density was here $410.0 \mathrm{~kg} / \mathrm{m}^{3}$. The density at the time when the pressure starts to increase is about $1600 \mathrm{~kg} / \mathrm{m}^{3}$. The simulation is stopped at the pressure of $100 \mathrm{MPa}$ after a calculation time of about 614 hours on 8 processors. The difference between the model with 110 and 200 spheres probably results from the different distributions of the grains. The distribution 
Table 2. Initial parameters for the sensitivity study.

\begin{tabular}{llll}
\hline Name & Number of spheres & mass $[\mathrm{kg}]$ & initial density $\left[\mathrm{kg}{ }^{\beta}\right]$ \\
\hline sand1 & 105 & $0.9297 \mathrm{E}-03$ & $0.9297 \mathrm{E}+03$ \\
sand2 & 98 & $0.8521 \mathrm{E}-03$ & $0.8521 \mathrm{E}+03$ \\
sand3 & 95 & $0.8632 \mathrm{E}-03$ & $0.8632 \mathrm{E}+03$ \\
sand4 & 103 & $0.9304 \mathrm{E}-03$ & $0.9304 \mathrm{E}+03$ \\
\hline
\end{tabular}

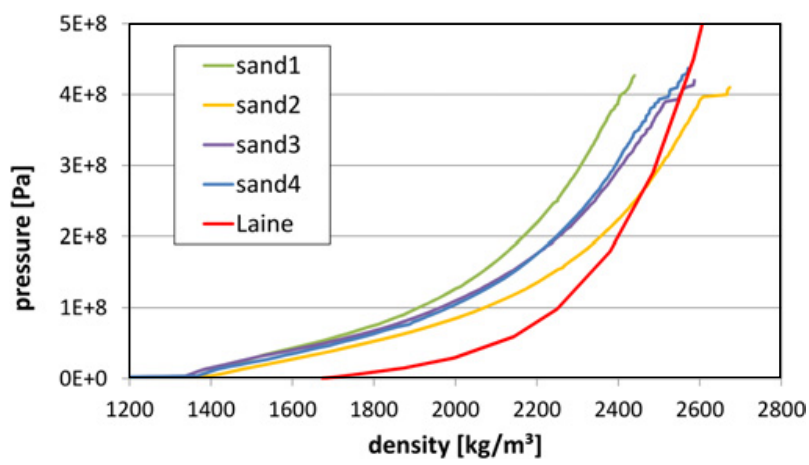

Fig. 8. Pressure-density curve of the numerical experiment (sensitivity study). Comparison with the data from Laine [1].

of the grains is chosen randomly. In addition, the virtual box of the latter model is larger, and, therefore, the initial density is smaller. The grains were configured from a lower initial density by hydrostatic pressure and not by the preprocessor.

\subsection{Sensitivity study with models of about 100 particles}

The influence of several random configurations of the same input variables of the preprocessor is determined using virtual boxes of $1 \mathrm{~cm}^{3}$. The initial parameters are given in Table 2 . The grading is simplified by using only particles with a diameter of $2.0 \mathrm{~mm}$ in order to minimize the number of parameters.

The resulting pressure-density curves are shown in Figure 8. It can be observed that the differences between the curves are small and that the influence of the configurations of the spheres are small.

\subsection{Unloading}

The experiments from Laine are performed in a way that the loading is increased stepwise and that unloading until a pressure of $0 \mathrm{~Pa}$ is performed between the load steps (Figure 9). The stiffness of the loading and unloading path is similar. This stiffness is increasing for increasing pressure level. A similar numerical experiment is performed using 25 particles (11648 elements) with a grading according to Table 1 . The numerical results are shown in Figure 9. Since the unloaded density of the experiments and of the numerical simulation are different, the density is shifted in Figure 9 of about $575 \mathrm{~kg} / \mathrm{m}^{3}$ to the left to allow a better comparison. It can be shown that the numerical behaviour corresponds with the experimental one.

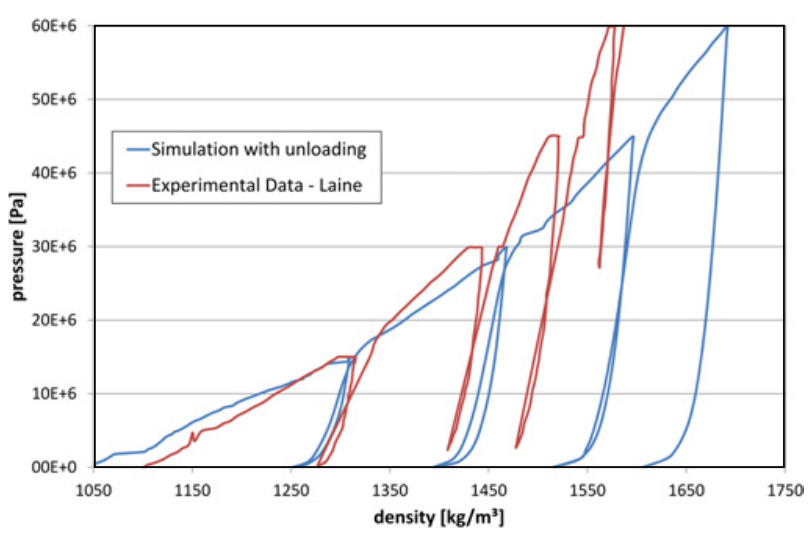

Fig. 9. Pressure-density curve of the numerical experiment (unloading, shift of $575 \mathrm{~kg} / \mathrm{m}^{3}$ to the left). Comparison with the data from Laine [1].

\section{Conclusions and outlook}

This work presents a method to obtain the pressure-density curve for granular materials like sand and gravel by using micromechanical models. The simulation model is built by a preprocessor which first develops a geometrical representation of sand or gravel by spheres and second creates from that a finite element model. This model is loaded hydrostatically. Various models are presented which are compared to the experimental data. The lack of corresponding experimental data including the grading of the material does not allow a detailed verification of the results.

The model presented can be used to obtain the influences of different gradings on the pressure-density curve. Another open question is the influence of the humidity on the hydrostatical behaviour of granular material. Full fluid-structure interaction in combination with water that is randomly distributed by the preprocessor should allow to determine the influence of humidity.

\section{References}

1. P. Laine, A. Sandvik, Derivation of Mechanical Properties for Sand, in Proceedings of the 4th Asia-Pacific Conference on Shock and Impact Loads on Structures (CI-Premier PTE LTD, Singapore, November 2001), pp. 361-368

2. N. Gebbeken, M. Ruppert, Archive of Applied Mechanics 70, 463 (2000)

3. M. Larcher, International Journal for Impact Engineering 36(5), 700 (2009)

4. D. Fiserova, Ph.D. thesis, Cranfield University (2006), http://hdl . handle.net/1826/1209

5. M. Grujicic, B. Pandurangan, R. Qiao, B. Cheeseman, W. Roy, R. Skaggs, R. Gupta, Soil Dynamics and Earthquake Engineering 28(1), 20 (2008)

6. M. Grujicic, B. Pandurangan, N. Coutris, B. Cheeseman, W. Roy, R. Skaggs, Soil Dynamics and Earthquake Engineering 28(12), 1045 (2008)

7. T. Hartmann, A. Pietzsch, G. N., International Journal of Protective Structures 1(4), 443 (2010)

8. W. Riedel, M. Wicklein, K. Thoma, International Journal of Impact Engineering 35(3), 155 (2008) 